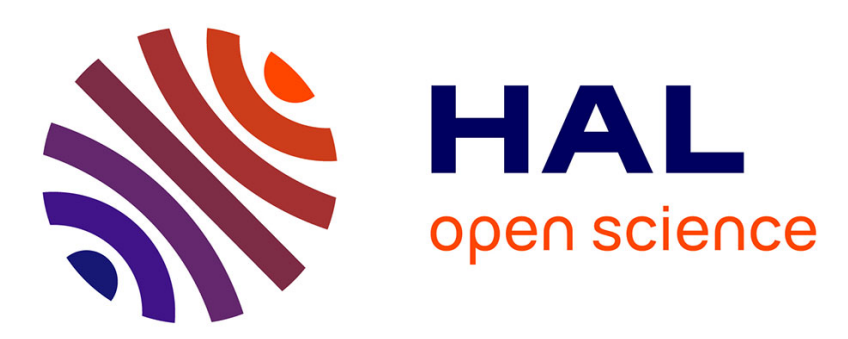

\title{
Improved backward error bounds for LU and Cholesky factorizations
}

\author{
Siegfried M. Rump, Claude-Pierre Jeannerod
}

\section{To cite this version:}

Siegfried M. Rump, Claude-Pierre Jeannerod. Improved backward error bounds for LU and Cholesky factorizations. 2013. hal-00841361v1

\section{HAL Id: hal-00841361 https://hal.inria.fr/hal-00841361v1}

Preprint submitted on 4 Jul 2013 (v1), last revised 23 Apr 2014 (v2)

HAL is a multi-disciplinary open access archive for the deposit and dissemination of scientific research documents, whether they are published or not. The documents may come from teaching and research institutions in France or abroad, or from public or private research centers.
L'archive ouverte pluridisciplinaire $\mathbf{H A L}$, est destinée au dépôt et à la diffusion de documents scientifiques de niveau recherche, publiés ou non, émanant des établissements d'enseignement et de recherche français ou étrangers, des laboratoires publics ou privés. 


\title{
IMPROVED BACKWARD ERROR BOUNDS FOR LU AND CHOLESKY FACTORIZATIONS
}

\author{
SIEGFRIED M. RUMP* AND CLAUDE-PIERRE JEANNEROD ${ }^{\dagger}$
}

\begin{abstract}
Assuming standard floating-point arithmetic (in base $\beta$, precision $p$ ) and barring underflow and overflow, classical rounding error analysis of the LU or Cholesky factorization of an $n \times n$ matrix $A$ provides backward error bounds of the form $|\Delta A| \leqslant \gamma_{n}|\widehat{L}||\widehat{U}|$ or $|\Delta A| \leqslant \gamma_{n+1}\left|\widehat{R}^{T}\right||\widehat{R}|$. Here, $\widehat{L}, \widehat{U}$, and $\widehat{R}$ denote the computed factors, and $\gamma_{n}$ is the usual fraction $n u /(1-n u)=n u+\mathcal{O}\left(u^{2}\right)$ with $u$ the unit roundoff. Similarly, when solving an $n \times n$ triangular system $T x=b$ by substitution, the computed solution $\widehat{x}$ satisfies $(T+\Delta T) \widehat{x}=b$ with $|\Delta T| \leqslant \gamma_{n}|T|$. All these error bounds contain quadratic terms in $u$ and limit $n$ to satisfy either $n u<1$ or $(n+1) u<1$. We show in this paper that the constants $\gamma_{n}$ and $\gamma_{n+1}$ can be replaced by $n u$ and $(n+1) u$, respectively, and that the restrictions on $n$ can be removed.

To get these new bounds the main ingredient is a general framework for bounding expressions of the form $|\rho-s|$, where $s$ is the exact sum of a floating-point number and $n-1$ real numbers, and where $\rho$ is a real number approximating the computed sum $\widehat{s}$. By instantiating this framework with suitable values of $\rho$, we obtain improved versions of the well-known Lemma 8.4 in Higham's ASNA [3, p. 142] (used for analyzing triangular system solving and LU factorization) and of its Cholesky variant [3, solution to Problem 10.3]. All our results hold for rounding to nearest with any tie-breaking strategy and no matter what the order of summation.
\end{abstract}

Key words. floating-point summation, rounding error analysis, backward error, LU factorization, Cholesky factorization, triangular system solving.

1. Introduction. Classical algorithms for triangularizing $n \times n$ matrices or solving $n \times n$ triangular systems consist of repeatedly evaluating expressions $y$ of the form

$$
y \in\left\{s, s / b_{k}, \sqrt{s}\right\}, \quad s=c-\sum_{i=1}^{k-1} a_{i} b_{i}, \quad k \leqslant n,
$$

for some scalars $a_{i}, b_{i}, c$. Specifically, the patterns $s$ and $s / b_{k}$ appear in Gaussian elimination for LU factorization or when solving triangular linear systems by substitution, while $s / b_{k}$ and $\sqrt{s}$ are the expressions typically used by Cholesky factorization.

One way to analyze the behavior of such algorithms in floating-point arithmetic is thus to bound the rounding errors committed during the evaluation of $y$ in (1.1a). Given a standard set $\mathbb{F}$ of floating-point numbers, assume that

$$
c \text { and all the } a_{i} \text { and } b_{i} \text { are in } \mathbb{F}
$$

and let $\widehat{y}$ be the result of a floating-point evaluation of $y$. For such $\widehat{y}$ backward error results are well known, and their central role in numerical linear algebra is made clear in Higham's book ASNA [3], where Lemma 8.4 covers the case $y \in\left\{s, s / b_{k}\right\}$ and Problem 10.3 considers the case $y=\sqrt{s}$. Writing $u$ for the unit roundoff associated with $\mathbb{F}$ and writing $\theta_{\ell}$ for reals such that

$$
\left|\theta_{\ell}\right| \leqslant \gamma_{\ell}:=\frac{\ell u}{1-\ell u} \quad \text { if } \ell u<1
$$

\footnotetext{
*Institute for Reliable Computing, Hamburg University of Technology, Schwarzenbergstraße 95, Hamburg 21071, Germany, and Faculty of Science and Engineering, Waseda University, 3-4-1 Okubo, Shinjuku-ku, Tokyo 169-8555, Japan (rump@tuhh.de).

${ }^{\dagger}$ INRIA, Laboratoire LIP (CNRS, ENS de Lyon, INRIA, UCBL), Université de Lyon, 46 allée d'Italie 69364 Lyon cedex 07, France (claude-pierre.jeannerod@ens-lyon.fr).
} 
these backward error results can be put in concise form as follows. ${ }^{1}$

If $y=s$ then

$$
\widehat{y}\left(1+\theta_{k-1}\right)=c-\sum_{i=1}^{k-1} a_{i} b_{i}\left(1+\theta_{k-1}^{(i)}\right) .
$$

If $y=s / b_{k}$ then

$$
b_{k} \widehat{y}\left(1+\theta_{k}\right)=c-\sum_{i=1}^{k-1} a_{i} b_{i}\left(1+\theta_{k}^{(i)}\right)
$$

If $y=\sqrt{s}$ then

$$
\widehat{y}^{2}\left(1+\theta_{k+1}\right)=c-\sum_{i=1}^{k-1} a_{i} b_{i}\left(1+\theta_{k+1}^{(i)}\right)
$$

Each of the three identities above holds no matter in what order of evaluation $\widehat{y}$ was produced, provided that no underflow or overflow occurred. Formally, no matter what the order of evaluation means to compute the sum using any of the rooted binary trees whose $k$ leaves are $c$ and the $a_{i} b_{i}$, and whose $k-1$ inner nodes have degree two and contain one intermediate sum each. For example, when $k=4$ amongst the possible schemes defined by such trees are

$\left(\left(c-a_{1} b_{1}\right)-a_{2} b_{2}\right)-a_{3} b_{3}, \quad\left(c-a_{1} b_{1}\right)-\left(a_{2} b_{2}+a_{3} b_{3}\right), \quad c-\left(\left(a_{1} b_{1}+a_{2} b_{2}\right)+a_{3} b_{3}\right)$.

Note also that for each of the equations in (1.3) the scalar $c$ is kept unperturbed. With $c$ playing the role of a given entry of a matrix over $\mathbb{F}$, this makes it possible to obtain backward error bounds for the LU or Cholesky factors of that matrix. Indeed, as shown in [3, Theorem 9.3], if for some $A \in \mathbb{F}^{m \times n}$ with $m \geqslant n$ Gaussian elimination runs to completion, then (1.3a-b) imply that the computed factors $\widehat{L}$ and $\widehat{U}$ satisfy

$$
\widehat{L} \widehat{U}=A+\Delta A, \quad|\Delta A| \leqslant \gamma_{n}|\widehat{L}||\widehat{U}|,
$$

with $\gamma_{n}$ as in (1.2) and where the absolute values and inequalities are to be understood componentwise. Similarly, if for some symmetric $A \in \mathbb{F}^{n \times n}$ Cholesky decomposition runs to completion, then (1.3b-c) imply that the computed factor $\widehat{R}$ satisfies

$$
\widehat{R}^{T} \widehat{R}=A+\Delta A, \quad|\Delta A| \leqslant \gamma_{n+1}\left|\widehat{R}^{T}\right||\widehat{R}| ;
$$

see [3, Theorem 10.3]. Furthermore, if $T x=b$ is solved by substitution for $T \in \mathbb{F}^{n \times n}$ triangular and nonsingular and $b \in \mathbb{F}^{n}$, then [3, Theorem 8.5] shows, by using (1.3b) and with $c$ now playing the role of an entry of the right-hand side $b$, that the computed solution $\widehat{x}$ satisfies

$$
(T+\Delta T) \widehat{x}=b, \quad|\Delta T| \leqslant \gamma_{n}|T| .
$$

\footnotetext{
${ }^{1}$ Hereafter, superscripts are used to indicate that the $\theta_{\ell}^{(i)}$ may be pairwise distinct and also distinct from $\theta_{\ell}$. In addition, the terms $\theta_{k}^{(i)}$ and $\theta_{k+1}^{(i)}$ are not best possible and can both be replaced by $\theta_{k-1}^{(i)}$. This is easily deduced from Higham's analysis in [3, pp. 141, 553] but does not impact the resulting backward error bounds for triangular system solving and LU and Cholesky factorizations; the reason is that such bounds are governed by the terms $\theta_{k}$ and $\theta_{k+1}$ in the left-hand sides of $(1.3 \mathrm{~b})$ and $(1.3 \mathrm{c})$.
} 
When $T$ is unit triangular, no division occurs during substitution and the constant $\gamma_{n}$ can be reduced to $\gamma_{n-1}$ by applying (1.3a) instead of (1.3b).

All identities in (1.3) are of the form

$$
\rho\left(1+\theta_{k+\ell-1}\right)=c-\sum_{i=1}^{k-1} a_{i} b_{i}\left(1+\theta_{k+\ell-1}^{(i)}\right)
$$

for suitable values of $\rho$ and $\ell$ : depending on the expression taken by $y$ in (1.1a), the real number $\rho$ is either $\widehat{y}, b_{k} \widehat{y}$, or $\widehat{y}^{2}$, and the integer $\ell$ is either 0,1 , or 2 . In any case, $\rho$ is (an approximation of) the computed value of the sum $s$ as in (1.1). Furthermore, due to (1.2) this generic backward error result is equivalent to the forward error bound

$$
|\rho-s| \leqslant \gamma_{k+\ell-1}\left(|\rho|+\sum_{i=1}^{k-1}\left|a_{i} b_{i}\right|\right) .
$$

Note in particular that this bound contains a quadratic term in $u$ and holds only if the condition $(k+\ell-1) u<1$ is satisfied.

In this paper we improve the bound (1.5), and thus the bounds in (1.3) and (1.4) as well, by showing that the constant $\gamma_{k+\ell-1}$ can be replaced by the unconditional and $\mathcal{O}\left(u^{2}\right)$-free value $(k+\ell-1) u$. To do so, we introduce a more general result. Instead of considering only special inner products $c-\sum_{i=1}^{k-1} a_{i} b_{i}$ as in (1.1), we allow the $a_{i} b_{i}$ to be replaced by $k-1 \leqslant n-1$ arbitrary real numbers. In other words, we consider general sums of the form

$$
s=x_{1}+\cdots+x_{n}, \quad x_{i} \in \mathbb{R} \text { for all } i \neq j \text { and } x_{j} \in \mathbb{F} \text { for some fixed } j .
$$

Writing $\mathrm{fl}\left(x_{i}\right)$ to denote an element of $\mathbb{F}$ nearest to $x_{i}$, let $\widehat{s}$ be the result of the sum $\mathrm{fl}\left(x_{1}\right)+\cdots+\mathrm{fl}\left(x_{n}\right)$ obtained in floating-point arithmetic, with rounding to nearest and no matter what the tie-breaking rule and what the order of evaluation. Furthermore, let $\rho$ be a real number approximating $\widehat{s}$ as

$$
|\rho-\widehat{s}| \leqslant \ell u|\rho| \quad \text { for some } \ell \in \mathbb{N} .
$$

Then, we show that in the absence of underflow and overflow,

$$
|\rho-s| \leqslant(n+\ell-1) u\left(|\rho|+\sum_{i=1, i \neq j}^{n}\left|x_{i}\right|\right) .
$$

This result holds without any restriction on $n$ and does not involve quadratic terms in $u$. Since $n u \leqslant \gamma_{n}$, it implies in particular (1.5).

By specializing (1.7) with suitable values of $\rho$ and $\ell$, we also obtain the following improved versions of the classical backward error results (1.3) and (1.4): First, for each $\theta_{\ell}$ (and $\theta_{\ell}^{(i)}$ ) appearing in (1.3) we can take

$$
\left|\theta_{\ell}\right| \leqslant \ell u \text { for all } \ell \text {, }
$$

instead of $\left|\theta_{\ell}\right| \leqslant \gamma_{\ell}$ for $\ell u<1$ as in (1.2). In other words, Higham's Lemma 8.4 and its Cholesky variant hold with the $\gamma_{\ell}$ terms replaced by $\ell u$ and with no restriction on $\ell$. Second, using these improved versions of the bounds in (1.3) we show that for LU 
and Cholesky factorizations and triangular system solving, the upper bounds in (1.4) can be replaced, respectively, by

$$
n u|\widehat{L}||\widehat{U}|, \quad(n+1) u\left|\widehat{R}^{T}\right||\widehat{R}|, \quad n u|T| \quad \text { for all } n .
$$

As long as neither underflow nor overflow occurs, each of these new backward error bounds holds in standard floating-point arithmetic with rounding to nearest, for any tie-breaking rule, any dimension, and any evaluation order of the sums involved.

The rest of this paper is organized as follows. Section 2 provides the necessary definitions and properties about floating-point arithmetic as well as two recent error bounds on floating-point summation. The proof of the bound (1.7) is then given in Section 3. In this section we also present some specializations of this bound that will be used later to refine (1.3). Furthermore, in the particular situation where $(\rho, \ell)=(\widehat{s}, 0)$ and all the $x_{i}$ are in $\mathbb{F}$ and accumulated recursively one after the other, we remark that we also have

$$
|\widehat{s}-s| \leqslant(n-1) u\left(|\widehat{s}|+\sum_{i=3}^{n}\left|x_{i}\right|\right) .
$$

Applications of (1.7) are then detailed in Section 4. We start with our improved version of Higham's Lemma 8.4 and its direct application to triangular system solving, and then turn to the improved backward error bounds for LU and Cholesky factorizations. Some concluding remarks in Section 5 finish the paper.

\section{Preliminaries.}

2.1. Floating-point numbers and rounding to nearest. Throughout this paper $\mathbb{F}$ denotes a set of finite floating-point numbers similar to those defined in the IEEE 754-2008 standard [4], with base $\beta$, precision $p$, and exponent range $\left[e_{\min }, e_{\max }\right]$. In particular, $\beta \geqslant 2, p \geqslant 1$, and $\mathbb{F}$ is symmetric and contains zero. (If $p=1$ then every element of $\mathbb{F}$ is either zero or an integer power of the base.)

A nonzero number in $\mathbb{F}$ is normal if its magnitude is at least $\beta^{e_{\min }}$, and subnormal otherwise. Accordingly, writing $\Omega$ for the largest number in $\mathbb{F}$, we say that a real number $t$ lies in the normal range of $\mathbb{F}$ if $\beta^{e_{\min }} \leqslant|t| \leqslant \Omega$, and that it lies in the subnormal range of $\mathbb{F}$ if $0<|t|<\beta^{e_{\min }}$.

We associate with the set $\mathbb{F}$ a round-to-nearest function $f$, which can be any map from $\mathbb{R}$ to $\mathbb{F} \cup\{ \pm \infty\}$ such that for all $t \in \mathbb{R}$ and if no overflow occurs,

$$
|\mathrm{fl}(t)-t|=\min _{s \in \mathbb{F}}|s-t| .
$$

Consequently, no assumption is made on the way of breaking ties.

Here and hereafter, overflow is defined in the same way as in the IEEE 754-2008 standard $[4, \S 7.4]$. Concerning underflow, we follow Kahan in [6]: when rounding a real number $t$, we say that underflow occurs if $t$ is in the subnormal range of $\mathbb{F}$ without being in $\mathbb{F}$, that is, if

$$
0<|t|<\beta^{e_{\min }} \quad \text { and } \quad \mathrm{fl}(t) \neq t .
$$

In terms of the IEEE 754-2008 standard this corresponds precisely to the event when the underflow flag is raised, assuming default exception handling; see [4, §7.5]. With this definition floating-point addition cannot cause underflow, since the sum of two floating-point numbers is exact when it lies in the subnormal range of $\mathbb{F}$; see for example [2]. Concerning other operations, underflow or overflow can occur for multiplication and division, but not for square root. 
2.2. Basic properties. Assuming $\mathbb{F}$ and $f$ as above, we have the following wellknown properties. First, if neither underflow nor overflow occurs when rounding $t \in \mathbb{R}$ to $\mathrm{fl}(t)$, then the errors relative to $t$ and $\mathrm{fl}(t)$ are both bounded by the unit roundoff $u=\frac{1}{2} \beta^{1-p}$. In other words,

$$
\begin{aligned}
\mathrm{fl}(t) & =t\left(1+\delta_{1}\right), \quad\left|\delta_{1}\right| \leqslant u, \\
& =\frac{t}{1+\delta_{2}}, \quad\left|\delta_{2}\right| \leqslant u .
\end{aligned}
$$

Note that since $\beta$ and $p$ are positive integers, we always have $u \leqslant 1 / 2$. The relation in (2.1a) is commonly referred to as the standard model of floating-point arithmetic. It is used in [3] together with the variant (2.1b) to derive the classical backward error results we have mentioned in introduction.

Second, for two floating-point numbers $a$ and $b$ it is known that $|\mathrm{fl}(a+b)-(a+b)| \leqslant$ $\min \{|a|,|b|\}$ if no overflow occurs; see for example [9] as well as [3, p. 91]. By combining this bound with (2.1b) and the fact that $\mathrm{fl}(a+b)$ cannot cause underflow, we deduce the following: if $a, b \in \mathbb{F}$, then

$$
|\mathrm{fl}(a+b)-(a+b)| \leqslant \min \{u|\mathrm{H}(a+b)|,|a|,|b|\} \quad \text { in the absence of overflow. }
$$

A third set of properties is obtained by considering the notion of unit in the first place (ufp), which was introduced in [8] and provides refinements to (2.1): a real number $t$ being given, ufp $(t)$ is defined as zero if $t$ is zero and, otherwise, as the unique integer power of the base $\beta$ such that $u f p(t) \leqslant|t|<\beta$ ufp $(t)$. Hence

$$
\operatorname{ufp}(t) \leqslant|t|
$$

for all $t$, and if $t$ is nonzero then its ufp can be thought of as the weight of its first nonzero digit in base- $\beta$ representation. The functions ufp and fl are known to be related as follows (see for example (2.13) and (2.18) in [8]). On the one hand, for $t \in \mathbb{R}$ and in the absence of overflow,

$$
|\mathrm{fl}(t)|=(1+k \cdot 2 u) \text { ufp }(t) \quad \text { for some } k \in \mathbb{N} .
$$

This implies in particular that

$$
\mathrm{ufp}(t)=|\mathrm{fl}(t)| \quad \text { or } \quad(1+2 u) \operatorname{ufp}(t) \leqslant|\mathrm{fl}(t)| .
$$

On the other hand, in the absence of both underflow and overflow,

$$
|\mathrm{fl}(t)-t| \leqslant u \cdot \mathrm{ufp}(t) .
$$

Hence $\left|\delta_{1}\right|$ and $\left|\delta_{2}\right|$ in (2.1) admit the sharper bounds $u \cdot \operatorname{ufp}(t) /|t|$ and $u \cdot \operatorname{ufp}(t) /|\mathrm{fl}(t)|$, respectively.

\subsection{Previous results: a priori error bounds for floating-point sums.} When using floating-point arithmetic as just described to evaluate $s=x_{1}+\cdots+x_{n}$, two types of bounds on $|\widehat{s}-s|$ have been obtained recently. They hold for all $n$ and no matter what order of evaluation was used to produce the result $\widehat{s}$. First, if all the $x_{i}$ are in $\mathbb{F}$, then it was shown in $[5, \S 3]$ that in the absence of overflow

$$
\left|\widehat{s}-\sum_{i=1}^{n} x_{i}\right| \leqslant(n-1) u \sum_{i=1}^{n}\left|x_{i}\right| .
$$


This bound had already appeared in [7] in the special case of recursive summation, that is, when $\widehat{s}$ is obtained using the evaluation order $\left(\ldots\left(\left(x_{1}+x_{2}\right)+x_{3}\right)+\cdots\right)+x_{n}$. Second, if all the $x_{i}$ are in $\mathbb{R}$ and such that $\mathrm{fl}\left(x_{i}\right)$ does not underflow, then it was shown in $[5, \S 4]$ that in the absence of overflow

$$
\left|\widehat{s}-\sum_{i=1}^{n} x_{i}\right| \leqslant n u \sum_{i=1}^{n}\left|x_{i}\right| .
$$

Here, $\widehat{s}$ is obtained by rounding each $x_{i}$ to $\mathrm{fl}\left(x_{i}\right)$ and then adding all the $\mathrm{fl}\left(x_{i}\right)$ in any given order. By taking each $x_{i}$ to be of the form $a_{i} b_{i}$ with $a_{i}, b_{i}$ in $\mathbb{F}$, we see that this second bound covers in particular the case of inner products of floating-point vectors of length $n$.

The above bounds thus improve upon the classical ones given in [3, pp. 63, 82] for sums of floating-point numbers and inner products of floating-point vectors: the terms $(n-1) u$ and $n u$ replace the classical terms $\gamma_{n-1}$ and $\gamma_{n}$, and the restrictions on $n$ are removed.

In this paper we will need (2.1b) but also each of the results in $(2.2),(2.3)$, and (2.4). Specifically, (2.2) and (2.4) are used in the proof of Theorem 3.1, while the ufpbased properties given in (2.3) are used to establish the second part of Corollary 3.2.

3. Main results. We start by showing that if some $x_{j}$ is a floating-point number, then $\left|x_{j}\right|$ in the right-hand side of (2.4) can be replaced by $|\widehat{s}|$ and the term $n u$ by $(n-1) u$. The difficulty is that $|\widehat{s}|$ may be small, even zero. Moreover, we show that the computed sum $\widehat{s}$ can be replaced by some real number when increasing the constant $n-1$ appropriately.

Theorem 3.1. Given $n \in \mathbb{N}_{>0}$ and $j \in\{1, \ldots, n\}$, let $x_{1}, \ldots, x_{n} \in \mathbb{R}$ be such that $x_{j} \in \mathbb{F}$ and, for all $i \neq j, \mathrm{fl}\left(x_{i}\right)$ does not underflow. Let $\widehat{s}$ be a floating-point sum of $\mathrm{fl}\left(x_{1}\right), \ldots, \mathrm{f}\left(x_{n}\right)$ no matter what the order of evaluation, and let $\rho \in \mathbb{R}$ be such that

$$
|\rho-\widehat{s}| \leqslant \ell u|\rho| \quad \text { for some } \ell \in \mathbb{N} .
$$

Then, in the absence of overflow,

$$
\Delta:=\rho-\sum_{i=1}^{n} x_{i} \quad \text { satisfies } \quad|\Delta| \leqslant(n+\ell-1) u\left(|\rho|+\sum_{i=1, i \neq j}^{n}\left|x_{i}\right|\right) .
$$

Proof. The proof is by induction on $n$. For $n=1$ the assertion $\left|\rho-x_{1}\right| \leqslant \ell u|\rho|$ is true because $x_{1} \in \mathbb{F}$ implies $\widehat{s}=x_{1}$. For $n \geqslant 2$ we assume that the result is true up to $n-1$, and we fix one evaluation order of the summation. In the corresponding summation tree, let $s_{1} \in \mathbb{R}$ be the node where $x_{j} \in \mathbb{F}$ is added and let $\widehat{s}_{1} \in \mathbb{F}$ denote its rounded value, that is,

$$
\widehat{s}_{1}=\mathrm{fl}\left(s_{1}\right), \quad s_{1}=x_{j}+\widehat{s}_{2} .
$$

Here $\widehat{s}_{2}$ is the root of a summation tree adding the elements of $\left\{x_{i}: i \in I_{2}\right\}$ for some non-empty index set $I_{2} \subseteq\{1, \ldots, n\} \backslash\{j\}$. Define $I_{1}=\{1, \ldots, n\} \backslash I_{2}$ and $I_{1}^{\prime}=I_{1} \backslash\{j\}$, and let $n_{j}$ be the cardinality of $I_{j}$ for $j=1,2$. In particular, $1 \leqslant n_{1}, n_{2} \leqslant n-1$ and $n_{1}+n_{2}=n$. Furthermore, $\widehat{s}$ is by definition the root of a summation tree adding $x_{1}, \ldots, x_{n}$, but due to (3.1) it is also the root of a summation tree $\mathcal{T}$ adding the $n_{1}<n$ elements of $\left\{\widehat{s}_{1}\right\} \cup\left\{x_{i}: i \in I_{1}^{\prime}\right\}$. Abbreviating

$$
\Delta_{1}=\rho-\left(\widehat{s}_{1}+\sum_{i \in I_{1}^{\prime}} x_{i}\right) \quad \text { and } \quad \Delta_{2}=\widehat{s}_{2}-\sum_{i \in I_{2}} x_{i}
$$


we use (3.1) to write $\Delta=\Delta_{1}+\widehat{s}_{1}-s_{1}+\Delta_{2}$, so that

$$
|\Delta| \leqslant\left|\Delta_{1}\right|+\left|\widehat{s}_{1}-s_{1}\right|+\left|\Delta_{2}\right|
$$

Since $\widehat{s}_{1} \in \mathbb{F}$ and $n_{1}<n$, applying the induction assumption to the tree $\mathcal{T}$ gives

$$
\left|\Delta_{1}\right| \leqslant\left(n_{1}+\ell-1\right) u \delta_{1} \quad \text { for } \quad \delta_{1}=|\rho|+\sum_{i \in I_{1}^{\prime}}\left|x_{i}\right| .
$$

On the other hand, applying [5, Proposition 4.1] to the sum of reals $\sum_{i \in I_{2}} x_{i}$ gives

$$
\left|\Delta_{2}\right| \leqslant n_{2} u \delta_{2} \quad \text { for } \quad \delta_{2}=\sum_{i \in I_{2}}\left|x_{i}\right|
$$

Third, we can bound $\left|\widehat{s}_{1}-s_{1}\right|$ as follows. Applying (2.2) to $s_{1}$ in (3.1) gives $\left|\widehat{s}_{1}-s_{1}\right| \leqslant$ $\min \left\{u\left|\widehat{s}_{1}\right|,\left|\widehat{s}_{2}\right|\right\}$. Furthermore, the definitions of the $\Delta_{i}$ and $\delta_{i}$ in (3.2) and (3.4a-b) imply that $\left|\widehat{s}_{i}\right| \leqslant\left|\Delta_{i}\right|+\delta_{i}$ for $i=1,2$. Hence

$$
\left|\widehat{s}_{1}-s_{1}\right| \leqslant \min \left\{u\left|\Delta_{1}\right|+u \delta_{1},\left|\Delta_{2}\right|+\delta_{2}\right\} .
$$

From (3.3) and (3.4) and depending on the expression chosen to bound $\left|\widehat{s}_{1}-s_{1}\right|$, we deduce the following two bounds on $|\Delta|$ :

$$
|\Delta| \leqslant\left(n_{1}+\ell\right) u \delta_{1}+\left(n_{1}+\ell-1\right) u^{2} \delta_{1}+n_{2} u \delta_{2}
$$

and

$$
|\Delta| \leqslant\left(n_{1}+\ell-1\right) u \delta_{1}+2 n_{2} u \delta_{2}+\delta_{2} .
$$

Recall that $\ell \geqslant 0, n_{1}, n_{2} \geqslant 1$, and $n=n_{1}+n_{2}$. Due to the special shape of its $\mathcal{O}\left(u^{2}\right)$ term, the bound in (3.5) implies the desired bound $B:=\left(n_{1}+n_{2}+\ell-1\right) u\left(\delta_{1}+\delta_{2}\right)$ on $|\Delta|$ as soon as $u \delta_{1} \leqslant \delta_{2}$ or $\left(n_{1}+\ell-1\right) u \leqslant n_{2}-1$. In the remaining case where

$$
\delta_{2}<u \delta_{1} \quad \text { and } \quad n_{2}-1<\left(n_{1}+\ell-1\right) u \text {, }
$$

we prove the bound $B$ by using (3.6). Recalling that $u \leqslant 1$, we have $n_{2}-1<n_{1}+\ell-1$. This inequality is strict and involves only integers, so it is equivalent to $n_{2} \leqslant n_{1}+\ell-1$. Consequently, (3.6) leads to $|\Delta|<\left(n_{1}+\ell\right) u \delta_{1}+\left(n_{1}+n_{2}+\ell-1\right) u \delta_{2} \leqslant B$, as wanted. This completes the proof.

Two observations can be made about Theorem 3.1:

(i) When we say "in the absence of overflow," we mean that overflow occurs neither when rounding the $x_{i}$ to the $\mathrm{fl}\left(x_{i}\right)$ nor when summing up these rounded values.

(ii) By our definition, underflow occurs if a result is in the subnormal range and causes a rounding error. Thus, in Theorem 3.1 neither the element $x_{j}$ itself, which is in $\mathbb{F}$, nor the additions can cause underflow, but only the rounding of the reals $x_{i}$ for $i \neq j$. Assuming that such rounding does not underflow, however, is necessary. To see this we may use arguments similar to the ones given immediately after [5, Proposition 4.1]. For example, consider $x_{j}=0$ and for $i \neq j$ let $x_{i}>0$ be so small that $\mathrm{fl}\left(x_{i}\right)=0$. The computed sum $\widehat{s}$ is then equal to zero. Hence, choosing $\rho=\widehat{s}=0$, we have $|\Delta|=\sum_{i \neq j}\left|x_{i}\right|$, which is generally not upper bounded by $(n+\ell-1) u|\Delta|$. 
Theorem 3.1 provides a general framework capable of handling arbitrary approximations $\rho$ to the computed sum $\widehat{s}$ of the $x_{i}$. In the corollary below we specialize this result to the two cases needed to refine the backward error bounds given in $[3$, Chaps. $8,9,10]$. The first case is when $\rho$ is the exact product of $b$ and $\mathrm{fl}(\widehat{s} / b)$ for a nonzero floating-point number $b$, and the second case is when $\rho$ is the exact square of $\mathrm{fl}(\sqrt{\widehat{s}})$.

COROllary 3.2. Let $x_{1}, \ldots, x_{n}$ and $\widehat{s}$ be as in Theorem 3.1. Assuming underflow and overflow do not occur, we have the following two error bounds.

If $b \in \mathbb{F}$ is nonzero and $\widehat{y}=\mathrm{fl}(\widehat{s} / b)$, then

$$
\left|b \widehat{y}-\sum_{i=1}^{n} x_{i}\right| \leqslant n u\left(|b \widehat{y}|+\sum_{i=1, i \neq j}^{n}\left|x_{i}\right|\right) .
$$

If $\widehat{s}$ is nonnegative and $\widehat{y}=\mathrm{fl}(\sqrt{\widehat{s}})$, then

$$
\left|\widehat{y}^{2}-\sum_{i=1}^{n} x_{i}\right| \leqslant(n+1) u\left(\widehat{y}^{2}+\sum_{i=1, i \neq j}^{n}\left|x_{i}\right|\right) .
$$

Proof. If $\widehat{y}=\mathrm{fl}(\widehat{s} / b)$ without underflow and overflow, then (2.1b) implies $|b \widehat{y}-\widehat{s}| \leqslant$ $u|b \widehat{y}|$, so the first implication follows from applying Theorem 3.1 with $\rho=b \widehat{y}$ and $\ell=1$. To prove the second implication, it suffices to check that $\widehat{y}=\mathrm{fl}(\sqrt{\widehat{s}})$ implies

$$
\left|\widehat{y}^{2}-\widehat{s}\right| \leqslant 2 u \widehat{y}^{2}
$$

and then to use Theorem 3.1 with $\rho=\widehat{y}^{2}$ and $\ell=2$. To show that (3.7) holds, using only (2.1b) is not enough (see Appendix A) and we therefore resort to the following ufp-based analysis.

Let $y=\sqrt{\widehat{\widehat{S}}}$. Recall from Section 2.1 that neither underflow nor overflow can occur when setting $\widehat{y}=\mathrm{fl}(y)$, and recall from $(2.3 \mathrm{c})$ that $\mathrm{ufp}(y)=\widehat{y}$ or $(1+2 u) \mathrm{ufp}(y) \leqslant \widehat{y}$. If $\operatorname{ufp}(y)=\widehat{y}$ then $(2.3 \mathrm{a})$ and $(2.1 \mathrm{~b})$ imply $\widehat{y} \leqslant y \leqslant(1+u) \widehat{y}$. Thus, taking squares and using (2.3b), $u<2$, and the fact that $y^{2}=\widehat{s}$ is a floating-point number, we get

$$
\widehat{y}^{2} \leqslant y^{2} \leqslant(1+2 u) \widehat{y}^{2},
$$

from which (3.7) follows. If $(1+2 u) \operatorname{ufp}(y) \leqslant \widehat{y}$ then, rewriting $\left|\widehat{y}^{2}-y^{2}\right|$ as $(\widehat{y}+y)|\widehat{y}-y|$ and applying $(2.1 \mathrm{~b})$ and $(2.3 \mathrm{~d})$, we obtain

$$
\left|\widehat{y}^{2}-y^{2}\right| \leqslant(2+u) \widehat{y} \cdot u \operatorname{ufp}(y) \leqslant \frac{2+u}{1+2 u} u \widehat{y}^{2} \leqslant 2 u \widehat{y}^{2}
$$

Therefore, the bound in (3.7) holds in both cases, which completes the proof.

We conclude this section by noting that if all the $x_{i}$ are in $\mathbb{F}$ and the order of evaluation is fixed to recursive summation, then both $x_{1}$ and $x_{2}$ can be omitted in the error estimate and replaced by the computed sum $\widehat{s}$. This may come as a surprise as $x_{1}$ and $x_{2}$ may be arbitrarily large compared to $\widehat{s}$. However, this is only true if $x_{1}$ and $x_{2}$ are of similar magnitude and opposite signs, that means cancellation occurs in $x_{1}+x_{2}$. But in this case $\mathrm{fl}\left(x_{1}+x_{2}\right)=x_{1}+x_{2}$ by Sterbenz' lemma [10], so no rounding error occurs. ${ }^{2}$ Note that this argument breaks down if not both $x_{1}$ and $x_{2}$

\footnotetext{
${ }^{2}$ More precisely, in the absence of overflow, $\mathrm{fl}(a-b)=a-b$ whenever $a, b \in \mathbb{F}$ satisfy $a / 2 \leqslant b \leqslant 2 a$; see $[10$, p. 138] and [3, p. 45].
} 
are floating-point numbers (take for example $x_{1}=-1$ and $x_{2}=1+u$.) More precisely, the following theorem holds true, the proof of which is deferred to Appendix B.

Theorem 3.3. Given $n \in \mathbb{N}_{>0}$, let $x_{1}, \ldots, x_{n}$ be in $\mathbb{F}$ and let $\hat{s}$ be the result of the floating-point evaluation of $x_{1}+\cdots+x_{n}$ by means of recursive summation. Then, in the absence of overflow,

$$
\left|\widehat{s}-\sum_{i=1}^{n} x_{i}\right| \leqslant(n-1) u\left(|\widehat{s}|+\sum_{i=3}^{n}\left|x_{i}\right|\right) .
$$

4. Applications. We show in this section that Theorem 3.1 and Corollary 3.2 can be used to improve upon the following five backward error results from Higham's book ASNA [3]: Lemma 8.4, Theorem 8.5 (triangular system solving), Theorem 9.3 (LU factorization), solution to Problem 10.3 (Cholesky variant of Lemma 8.4), and Theorem 10.3 (Cholesky factorization).

Hereafter we write $a_{i j}$ to denote the $(i, j)$ entry of a matrix $A$, and $\operatorname{diag}(f(i))$ for the $n \times n$ diagonal matrix whose $(i, i)$ entry equals $f(i)$. Hence, whenever we use the diag notation there is an implicit assumption that the index $i$ ranges from 1 to $n$.

4.1. An improved version of Higham's Lemma 8.4. The results of Section 3 imply first that Higham's Lemma 8.4 [3, p. 142] can be rewritten as follows, with the original $\gamma_{k}$ and $\gamma_{k-1}$ terms replaced by $k u$ and $(k-1) u$ and with no restriction on $k$.

LEMMA 4.1. Let $k \in \mathbb{N}_{>0}$ and $a_{1}, \ldots, a_{k-1}, b_{1}, \ldots, b_{k-1}, b_{k}, c \in \mathbb{F}$ be given, with $b_{k}$ nonzero. If $y=\left(c-\sum_{i=1}^{k-1} a_{i} b_{i}\right) / b_{k}$ is evaluated in floating-point arithmetic then, in the absence of underflow and overflow and no matter what the order of evaluation, the computed $\widehat{y}$ satisfies

$$
b_{k} \widehat{y}\left(1+\theta_{k}^{(0)}\right)=c-\sum_{i=1}^{k-1} a_{i} b_{i}\left(1+\theta_{k}^{(i)}\right), \quad\left|\theta_{k}^{(i)}\right| \leqslant k u \text { for all } i
$$

If $b_{k}=1$, so that there is no division, then $\left|\theta_{k}^{(i)}\right| \leqslant(k-1) u$ for all $i$.

Proof. Assume first that $b_{k}=1$. In this case, by applying Theorem 3.1 with $\rho=\widehat{y}, \ell=0, n=k, x_{1}=c$ and $x_{i+1}=-a_{i} b_{i}$ for $1 \leqslant i<n$, we obtain

$$
|\widehat{y}-y| \leqslant(k-1) u\left(|\widehat{y}|+\sum_{i=1}^{k-1}\left|a_{i} b_{i}\right|\right)
$$

Hence $\widehat{y}-y=\epsilon\left(|\widehat{y}|+\sum_{i=1}^{k-1}\left|a_{i} b_{i}\right|\right)$ for some $\epsilon$ in $\mathbb{R}$ with $|\epsilon| \leqslant(k-1) u$. Defining $\epsilon^{(0)}=-\operatorname{sign}(\widehat{y}) \epsilon$ and $\epsilon^{(i)}=-\operatorname{sign}\left(a_{i} b_{i}\right) \epsilon$ for $1 \leqslant i<k$, we deduce that $\widehat{y}\left(1+\epsilon^{(0)}\right)=$ $c-\sum_{i=1}^{k-1} a_{i} b_{i}\left(1+\epsilon^{(i)}\right)$, where $\left|\epsilon^{(i)}\right|=|\epsilon| \leqslant(k-1) u$ for all $i$.

The general situation where $b_{k}$ is a nonzero floating-point number is handled analogously by using Corollary 3.2 with $b=b_{k}, n=k$, and the $x_{i}$ as above.

Lemma 4.1 then leads immediately to the following backward error result for triangular system solving and improves upon [3, Theorem 8.5]: given $b \in \mathbb{F}^{n}$ and $T \in \mathbb{F}^{n \times n}$ triangular and nonsingular, then, in the absence of underflow or overflow, substitution produces an approximate solution $\widehat{x}$ to $T x=b$ that satisfies

$$
(T+\Delta T) \widehat{x}=b, \quad|\Delta T| \leqslant \operatorname{diag}\left(d_{k}\right)|T| \leqslant n u|T|
$$


with

$$
d_{k}= \begin{cases}k u, & \text { if } T \text { is lower triangular, } \\ (n-k+1) u, & \text { if } T \text { is upper triangular. }\end{cases}
$$

If in addition $T$ is unit triangular, then $d_{k}$ can be decreased further to $(k-1) u$ or $(n-k) u$, and the constant $n u$ in the bound (4.1a) can be replaced by $(n-1) u$.

4.2. An improved backward error bound for LU factorization. We now turn to the computation of an LU factorization by means of any variant of Gaussian elimination, and give the following improvement to [3, Theorem 9.3].

Theorem 4.2. Let $A \in \mathbb{F}^{m \times n}$ with $m \geqslant n$. If Gaussian elimination runs to completion then, in the absence of underflow and overflow, the computed factors $\widehat{L} \in \mathbb{F}^{m \times n}$ and $\widehat{U} \in \mathbb{F}^{n \times n}$ satisfy

$$
\widehat{L} \widehat{U}=A+\Delta A, \quad|\Delta A| \leqslant n u|\widehat{L}||\widehat{U}| .
$$

If $m=n$ then sharper bounds are

$$
\begin{aligned}
|\Delta A| & \leqslant \operatorname{diag}((i-1) u)|\widehat{L}||\widehat{U}| \\
& \leqslant(n-1) u|\widehat{L}||\widehat{U}| .
\end{aligned}
$$

Proof. As shown by Higham in [3, pp. 162-163] it suffices to analyze one of the mathematically equivalent formulations of Gaussian elimination, for example, Doolittle's method: for $k=1, \ldots, n$ and given the first $k-1$ columns of $\widehat{L}$ and the first $k-1$ rows of $\widehat{U}$, the $k$ th row of $\widehat{U}$ and the $k$ th column of $\widehat{L}$ are obtained by floating-point evaluation of the expressions

$$
\begin{gathered}
y_{k j}=a_{k j}-\sum_{i=1}^{k-1} \widehat{\ell}_{k i} \widehat{u}_{i j}, \quad j=k, \ldots, n, \\
y_{i k}=\left(a_{i k}-\sum_{j=1}^{k-1} \widehat{\ell}_{i j} \widehat{u}_{j k}\right) / y_{k k}, \quad i=k+1, \ldots, m .
\end{gathered}
$$

Writing $\widehat{u}_{k j}$ and $\widehat{\ell}_{i k}$ for the computed values of $y_{k j}$ and $y_{i k}$ and setting $\widehat{\ell}_{k k}=1$, we deduce from Lemma 4.1 that, no matter what the order of evaluation,

$$
\begin{aligned}
&\left|a_{k j}-\sum_{i=1}^{k} \widehat{\ell}_{k i} \widehat{u}_{i j}\right| \leqslant(k-1) u \sum_{i=1}^{k}\left|\widehat{\ell}_{k i}\right|\left|\widehat{u}_{i j}\right|, \quad j=k, \ldots, n, \\
&\left|a_{i k}-\sum_{j=1}^{k} \widehat{\ell}_{i j} \widehat{u}_{j k}\right| \leqslant k u \sum_{j=1}^{k}\left|\widehat{\ell}_{i j}\right|\left|\widehat{u}_{j k}\right|, \quad i=k+1, \ldots, m .
\end{aligned}
$$

Since $k \leqslant n$, the inequalities in (4.3) imply $|A-\widehat{L} \widehat{U}| \leqslant n u|\widehat{L}||\widehat{U}|$. If in addition $m=n$ then the constant term $k u$ in $(4.3 \mathrm{~b})$ satisfies $k u \leqslant(i-1) u \leqslant(n-1) u$, which leads to the improved bounds $\operatorname{diag}((i-1) u)|\widehat{L}||\widehat{U}|$ and $(n-1) u|\widehat{L}||\widehat{U}|$.

4.3. An improved backward error bound for Cholesky factorization. If $A \in \mathbb{R}^{n \times n}$ is symmetric positive definite, its Cholesky factor is the unique upper triangular matrix $R \in \mathbb{R}^{n \times n}$ such that $A=R^{T} R$, and by Cholesky factorization we 
mean the computation of $R$ using the conventional algorithm, described for example in [3, Algorithm 10.2]. This algorithm requires to evaluate not only expressions of the form $s / b_{k}$ with $s=c-\sum_{i=1}^{k-1} a_{i} b_{i}$, but also expressions of the form $\sqrt{s}$. Thus we start with the following variant of Lemma 4.1.

LEMMA 4.3. Let $k \in \mathbb{N}_{>0}$ and $a_{1}, \ldots, a_{k-1}, b_{1}, \ldots, b_{k-1}, c \in \mathbb{F}$ be given, let $s=c-\sum_{i=1}^{k-1} a_{i} b_{i}$ be evaluated in floating-point arithmetic, and let $\widehat{s}$ denote the resulting approximation of $s$. In the absence of underflow and overflow and if $\hat{s}$ is nonnegative, then, no matter in what order of evaluation $\widehat{s}$ was produced, $\widehat{y}=\mathrm{fl}(\sqrt{\widehat{s}})$ satisfies

$$
\widehat{y}^{2}\left(1+\theta_{k+1}^{(0)}\right)=c-\sum_{i=1}^{k-1} a_{i} b_{i}\left(1+\theta_{k+1}^{(i)}\right), \quad\left|\theta_{k+1}^{(i)}\right| \leqslant(k+1) u \text { for all } i .
$$

Proof. This result follows from the second bound in Corollary 3.2 with $n=k$, $x_{1}=c$, and $x_{i+1}=-a_{i} b_{i}$ for $1 \leqslant i<n$.

Using only the standard models in (2.1), Higham shows in [3, solution to Problem 10.3] that the identity above holds with $\left|\theta_{k+1}^{(0)}\right| \leqslant \gamma_{k+1}$ and $\left|\theta_{k+1}^{(i)}\right| \leqslant \gamma_{k-1}$ for $i \geqslant 1$. His relative error bounds on the $a_{i} b_{i}$ are thus potentially sharper than ours. However, classically the backward error of Cholesky factorization is bounded by a single constant, namely

$$
\max _{0 \leqslant i<k}\left|\theta_{k+1}^{(i)}\right| \leqslant \gamma_{k+1}
$$

whereas Lemma 4.3 above gives

$$
\max _{0 \leqslant i<k}\left|\theta_{k+1}^{(i)}\right| \leqslant(k+1) u \quad \text { without restriction on } k \text {. }
$$

Combining Lemmas 4.1 and 4.3 leads to the following backward error bound for the Cholesky factor, which improves upon [3, Theorem 10.3].

TheOrem 4.4. Let $A \in \mathbb{F}^{n \times n}$ be symmetric. If Cholesky factorization runs to completion then, in the absence of underflow and overflow, the computed factor $\widehat{R} \in \mathbb{F}^{n \times n}$ satisfies

$$
\begin{aligned}
\widehat{R}^{T} \widehat{R}=A+\Delta A, \quad|\Delta A| & \leqslant \operatorname{diag}((i+1) u)\left|\widehat{R}^{T}\right||\widehat{R}| \\
& \leqslant(n+1) u\left|\widehat{R}^{T}\right||\widehat{R}| .
\end{aligned}
$$

Proof. The conventional method for Cholesky factorization computes $\widehat{R}$ one column at a time (cf. Algorithm 10.2 in [3]): for $j=1, \ldots, n$ and given the first $j-1$ columns of $\widehat{R}$, the $j$ th column is obtained by evaluating in floating-point the expressions

$$
\begin{gathered}
y_{i j}=\left(a_{i j}-\sum_{k=1}^{i-1} \widehat{r}_{k i} \widehat{r}_{k j}\right) / \widehat{r}_{i i}, \quad i=1, \ldots, j-1, \\
y_{j j}=\left(a_{j j}-\sum_{k=1}^{j-1} \widehat{r}_{k j}^{2}\right)^{1 / 2} .
\end{gathered}
$$


Let us denote by $\widehat{r}_{i j}$ and $\widehat{r}_{j j}$ the computed values of $y_{i j}$ and $y_{j j}$, respectively, no matter what the order of evaluation. Then, applying Lemma 4.1 and Lemma 4.3,

$$
\begin{gathered}
\left|a_{i j}-\sum_{k=1}^{i} \widehat{r}_{k i} \widehat{r}_{k j}\right| \leqslant i u \sum_{k=1}^{i}\left|\widehat{r}_{k i}\right|\left|\widehat{r}_{k j}\right|, \quad i=1, \ldots, j-1, \\
\left|a_{j j}-\sum_{k=1}^{j} \widehat{r}_{k j}^{2}\right| \leqslant(j+1) u \sum_{k=1}^{j} \widehat{r}_{k j}^{2} .
\end{gathered}
$$

The conclusion follows from $A-\widehat{R}^{T} \widehat{R}$ and $\widehat{R}^{T} \widehat{R}$ being symmetric.

5. Concluding remarks. The framework introduced in Theorem 3.1 leads to refined backward error bounds for triangular system solving and, perhaps more importantly, for LU and Cholesky factorizations. Thus, when solving a linear system $A x=b$ by means of such factorizations, it is natural to ask whether the classical backward error bounds for the computed solution $\widehat{x}$ can be improved as well. The answer is yes, but as we will see now, to a lesser extent. Classically, we have $(A+\Delta A) \widehat{x}=b$ with $|\Delta A| \leqslant \gamma_{3 n}|\widehat{L}||\widehat{U}|$ if LU factorization is used, and $|\Delta A| \leqslant \gamma_{3 n+1}\left|\widehat{R}^{T}\right||\widehat{R}|$ if Cholesky factorization is used; see Theorems 9.4 and 10.4 in [3]. By applying (4.1), (4.2) and (4.4) directly to the proof of these two theorems, improved bounds are easily obtained:

$$
\begin{aligned}
|\Delta A| & \leqslant \operatorname{diag}\left((n+2 i-2) u+n(i-1) u^{2}\right)|\widehat{L}||\widehat{U}| \\
& \leqslant\left((3 n-2) u+\left(n^{2}-n\right) u^{2}\right)|\widehat{L}||\widehat{U}|
\end{aligned}
$$

in the case of LU factorization, and

$$
\begin{aligned}
|\Delta A| & \leqslant \operatorname{diag}\left((n+2 i+1) u+n i u^{2}\right)\left|\widehat{R}^{T}\right||\widehat{R}| \\
& \leqslant\left((3 n+1) u+n^{2} u^{2}\right)\left|\widehat{R}^{T}\right||\widehat{R}|
\end{aligned}
$$

in the case of Cholesky factorization. These bounds hold for all $n$ and no matter what the order of evaluation; in addition, their constants are always smaller than the classical constants $\gamma_{3 n}$ and $\gamma_{3 n+1}$. However, in both cases a term quadratic in $u$ remains, and it is not clear whether it can be removed or not. If this is possible then further techniques than those introduced in this paper might be needed to achieve unconditional error bounds whose constants are $(3 n-2) u$ and $(3 n+1) u$.

But even if it were possible in the example above, it is important to realize that the terms $\gamma_{\ell}$ cannot generally be replaced by $\ell u$. Indeed, this already happens in the simple case of pairwise summation of floating-point numbers. When adding $n$ floating-point numbers $x_{1}, \ldots, x_{n}$ the classical analysis bounds the absolute error by $\gamma_{\ell} \sum_{i=1}^{n}\left|x_{i}\right|$, where $\ell$ is the height of the binary tree underlying the evaluation order $[1,3]$. For pairwise summation this tree has the minimum possible height, namely, $\ell=\left\lceil\log _{2} n\right\rceil$, but in this case the actual error can be larger than $\ell u \sum_{i=1}^{n}\left|x_{i}\right|$. To prove this, it suffices to consider the following construction. Let $n=2^{\ell}$, assume $\beta=2$, and let $x_{1}, \ldots, x_{n} \in \mathbb{F}$ be defined recursively as $x_{1}=1$ and $x_{n / 2+i}=u x_{i}$ for $i=1, \ldots, n / 2$. Then, for rounding to nearest with ties broken "to away," a term strictly larger than $\ell u$ is necessary for large enough $\ell$. Similar examples can be found for other tie-breaking rules. Although such counterexamples require a large exponent range and a huge dimension, they illustrate the impossibility, in general, to systematically replace $\gamma_{\ell}$ terms by $\ell u$. 
Appendix A. Proof that (2.1b) does not imply (3.7). Let $\mathbb{F}$ with $\beta=2$ and unit roundoff $u=2^{-p} \leqslant 1 / 2$ be given, and consider the set $\overline{\mathbb{F}}$ obtained from $\mathbb{F}$ by replacing 2 and -2 by $2+2 u+u^{2}$ and $-\left(2+2 u+u^{2}\right)$, respectively. Furthermore, let $\overline{\mathrm{fl}}$ denote a round-to-nearest function from $\mathbb{R}$ to $\overline{\mathbb{F}} \cup\{ \pm \infty\}$.

In the set $\mathbb{F}$, the predecessor and successor of 2 are $2-2 u$ and $2+4 u$, and $u$ is small enough to ensure $2+2 u+u^{2} \leqslant 2+4 u$. Therefore, $2-2 u$ and $2+2 u+u^{2}$ are two consecutive elements of $\overline{\mathbb{F}}$, and their midpoint, which lies exactly halfway between them, is

$$
\mu=2+\frac{1}{2} u^{2}
$$

Given a real number $t$ in the normal range of $\overline{\mathbb{F}}$ (which is identical to the normal range of $\mathbb{F}$ ), the rounding of $t$ to a nearest element of $\overline{\mathbb{F}}$ is modeled as follows: if $2-2 u<|t|<2+2 u+u^{2}$ then the error relative to $\overline{\mathrm{fl}}(t)$ satisfies

$$
\frac{|\overline{\mathrm{f}}(t)-t|}{|\overline{\mathrm{f}}(t)|} \leqslant \frac{\mu}{2-2 u}-1=u \frac{1+\frac{1}{4} u}{1-u}=: \bar{u}
$$

and otherwise it is bounded by $u$. Since $u \leqslant \bar{u}$, it follows that the model $(2.1 \mathrm{~b})$ holds with $\mathbb{F}, \mathrm{fl}, u$ replaced by $\overline{\mathbb{F}}, \overline{\mathrm{f}}, \bar{u}$.

Now, let $\widehat{s}=4$ and $\widehat{y}=\overline{\mathrm{fl}}(\sqrt{\widehat{s}})$. We see that $\widehat{s}$ belongs to $\overline{\mathbb{F}}$ and, since $2-2 u \leqslant$ $2<\mu$, we have $\widehat{y}=2-2 u$. (Note that this is true no matter to which of its neighbors the midpoint $\mu$ is rounded.) Hence, on the one hand,

$$
\left|\widehat{y}^{2}-\widehat{s}\right|=8 u-4 u^{2}
$$

and, on the other hand,

$$
2 \bar{u} \widehat{y}^{2}=8 u-6 u^{2}-2 u^{3} .
$$

Consequently, $\left|\widehat{y}^{2}-\widehat{s}\right|>2 \bar{u} \widehat{y}^{2}$, that is, (3.7) does not hold for $\overline{\mathbb{F}}, \overline{\mathrm{f}}, \bar{u}$. This concludes the proof that assuming only (2.1b) is not enough to ensure (3.7).

Appendix B. Proof of Theorem 3.3. The proof is by induction on $n$ and borrows some ideas from [7]. The case $n=1$ is trivial and the case $n=2$ corresponds to the second standard model, given in $(2.1 \mathrm{~b})$. Let us now assume that $n \geqslant 3$ and that the result is true up to $n-1$. Writing $\widehat{s}_{1}=x_{1}$, we start by defining for $2 \leqslant i \leqslant n$

$$
s_{i}=\widehat{s}_{i-1}+x_{i}, \quad \widehat{s}_{i}=\mathrm{fl}\left(s_{i}\right), \quad \Delta_{i}=\widehat{s}_{i}-\left(x_{1}+\cdots+x_{i}\right) .
$$

With this notation our goal is to show that $\left|\Delta_{n}\right| \leqslant(n-1) u\left(\left|\widehat{s}_{n}\right|+\sum_{i=3}^{n}\left|x_{i}\right|\right)$.

It is easily verified from (B.1) that for $2 \leqslant j<n$ the following relations hold:

$$
\Delta_{n}=\sum_{i=j+1}^{n}\left(\widehat{s}_{i}-s_{i}\right)+\Delta_{j} \quad \text { and } \quad \widehat{s}_{j}=\sum_{i=j+1}^{n}\left(s_{i}-\widehat{s}_{i}-x_{i}\right)+\widehat{s}_{n} .
$$

Let us first use (B.2) with $j=n-1$. The identity on the left gives

$$
\begin{aligned}
\left|\Delta_{n}\right| & \leqslant\left|\widehat{s}_{n}-s_{n}\right|+\left|\Delta_{n-1}\right| \\
& \leqslant u\left|\widehat{s}_{n}\right|+(n-2) u\left(\left|\widehat{s}_{n-1}\right|+\sum_{i=3}^{n-1}\left|x_{i}\right|\right),
\end{aligned}
$$


by using $(2.1 \mathrm{~b})$ together with the induction hypothesis. Since the right identity in (B.2) and (2.1b) also lead to $\left|\widehat{s}_{n-1}\right| \leqslant\left|\widehat{s}_{n}-s_{n}\right|+\left|x_{n}\right|+\left|\widehat{s}_{n}\right| \leqslant(1+u)\left|\widehat{s}_{n}\right|+\left|x_{n}\right|$, we arrive at

$$
\left|\Delta_{n}\right| \leqslant(n-1) u\left|\widehat{s}_{n}\right|+(n-2) u^{2}\left|\widehat{s}_{n}\right|+(n-2) u \sum_{i=3}^{n}\left|x_{i}\right| .
$$

Let us now use (B.2) with $j=2$. On the one hand,

$$
\begin{aligned}
\left|\Delta_{n}\right| & \leqslant \sum_{i=3}^{n}\left|\widehat{s}_{i}-s_{i}\right|+\left|\Delta_{2}\right| \\
& \leqslant \sum_{i=3}^{n}\left|x_{i}\right|+u\left|\widehat{s}_{2}\right|,
\end{aligned}
$$

since $\left|\widehat{s}_{i}-s_{i}\right| \leqslant\left|x_{i}\right|$ by (B.1) and (2.2) and since $\left|\Delta_{2}\right|=\left|\widehat{s}_{2}-s_{2}\right| \leqslant u\left|\widehat{s}_{2}\right|$ by (2.1b). On the other hand,

$$
\begin{aligned}
\left|\widehat{s}_{2}\right| & \leqslant \sum_{i=3}^{n}\left|s_{i}-\widehat{s}_{i}\right|+\sum_{i=3}^{n}\left|x_{i}\right|+\left|\widehat{s}_{n}\right| \\
& \leqslant 2 \sum_{i=3}^{n}\left|x_{i}\right|+\left|\widehat{s}_{n}\right| .
\end{aligned}
$$

Hence, from (B.4) and (B.5) and since by assumption $2 \leqslant n-1$,

$$
\left|\Delta_{n}\right| \leqslant u\left|\widehat{s}_{n}\right|+\sum_{i=3}^{n}\left|x_{i}\right|+(n-1) u \sum_{i=3}^{n}\left|x_{i}\right| .
$$

The desired bound $\left|\Delta_{n}\right| \leqslant(n-1) u\left(\left|\widehat{s}_{n}\right|+\sum_{i=3}^{n}\left|x_{i}\right|\right)$ then follows immediately from either (B.3) or (B.6), depending on how $(n-2) u\left|\widehat{s}_{n}\right|$ compares with $\sum_{i=3}^{n}\left|x_{i}\right|$. This concludes the proof of Theorem 3.3.

\section{REFERENCES}

[1] T. O. Espelid, On floating-point summation, SIAM Rev., 37 (1995), pp. 603-607.

[2] J. R. HAuser, Handling floating-point exceptions in numeric programs, ACM Trans. Program. Lang. Syst., 18 (1996), pp. 139-174.

[3] N. J. Higham, Accuracy and Stability of Numerical Algorithms, SIAM, Philadelphia, second ed., 2002.

[4] IEEE Computer Society, IEEE Standard for Floating-Point Arithmetic, IEEE Standard 754-2008, Aug. 2008. available at http://ieeexplore.ieee.org/servlet/opac?punumber= 4610933.

[5] C.-P. Jeannerod And S. M. Rump, Improved error bounds for inner products in floating-point arithmetic, SIAM J. Matrix Anal. Appl., 34 (2013), pp. 338-344.

[6] W. Kahan, A brief tutorial on gradual underflow, 2005. Available at http://www.cs.berkeley . edu/ wkahan/.

[7] S. M. RUMP, Error estimation of floating-point summation and dot product, BIT, 52 (2012), pp. 201-220.

[8] S. M. Rump, T. Ogita, And S. Oishi, Accurate floating-point summation, Part I: Faithful rounding, SIAM J. Sci. Comput., 31 (2008), pp. 189-224.

[9] J. R. ShewCHuk, Adaptive precision floating-point arithmetic and fast robust geometric predicates, Discrete and Computational Geometry, 18 (1997), pp. 305-363.

[10] P. H. Sterbenz, Floating-Point Computation, Prentice-Hall, Englewood Cliffs, NJ, USA, 1974. 